\title{
Letter to the Editor: "On exact recovery of sparse vectors from linear measurements" [Mathematical Notes 94 (1), 107-114 (2013)]
}

\author{
S. V. Konyagin ${ }^{*}$, Yu. V. Malykhin ${ }^{1}$, and K. S. Ryutin ${ }^{2}$ \\ ${ }^{1}$ Steklov Mathematical Institute, Moscow, Russia \\ ${ }^{2}$ Moscow State University, Moscow, Russia \\ Received December 23, 2013
}

DOI: $10.1134 / \mathrm{S} 000143461409020 \mathrm{X}$

As we learned from a lecture by Professor Ullrich some time after the publication of our paper [1], Theorem 1 contained there is not new. The corresponding result is contained in Lemma 2.4 from [2]. Somewhat earlier, a similar construction was used to obtain similar results in [3] (see Theorem 1.1 there).

The proofs are based on similar considerations. More precisely, in our paper, the problem of the uniqueness of the recovery of a sparse vector from linear measurements, using duality, was reduced to the question of the values of $n, N, k$ for which the following inequality holds:

$$
d_{n}\left(V_{k}^{N}, \ell_{\infty}^{N}\right) \geq \frac{1}{2}
$$

where $d_{n}$ is the Kolmogorov width and

$$
V_{k}^{N}=\left\{\left(v_{1}, \ldots, v_{N}\right): v_{j} \in\{-1,0,1\}, \sum_{j=1}^{N}\left|v_{j}\right|=k\right\} .
$$

Further, we evaluated this width using a combinatorial construction and duality considerations.

The authors of [2] used a combinatorial construction similar to ours and obtained the required result directly from the recovery uniqueness condition. For them, this result is, to some extent, an auxiliary one, while their main results deal with the estimation of the Gelfand width $d^{m}\left(B_{p}^{N}, l_{q}^{N}\right), 0<p \leq 1, p<q \leq 2$, and their estimates are almost sharp.

The question of the estimation of the Kolmogorov widths $d_{n}\left(V_{k}^{N}, \ell_{\infty}^{N}\right)$ was not considered in [2].

The relationship between the problem of the recovery of sparse vectors from linear measurements and the estimates of various widths was described in great detail in the recent paper [4].

\section{REFERENCES}

1. S. V. Konyagin, Yu. V. Malykhin, and K. S. Ryutin, Math. Notes 94 (1), 107-114 (2013).

2. S. Foucart, A. Pajor, H. Rauhut, and T. Ullrich, J. Complexity 26 (6), 629 (2010).

3. N. Linial and I. Novik, Discrete Comput. Geom. 36 (2), 273 (2006).

4. A. Pinkus, J. Approx. Theory 163 (3), 388 (2011).

\footnotetext{
*E-mail: konyagin23@gmail.com, konyagin@mi.ras.ru
} 